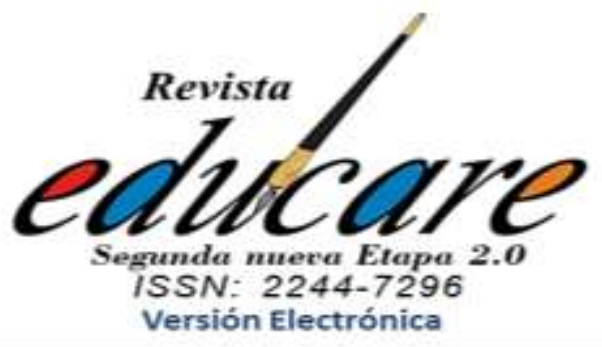

Volumen $25 \mathrm{~N}^{\circ} 1$ Enero - Abril 2021
$(421-437)$

\section{EL CUENTO INFANTIL COMO ESTRATEGIA PARA POTENCIAR EL LENGUAJE EN NIÑOS CON AUTISMO}

\section{Alicia Jaqueline Álvarez Mérida}

ORCID: https://orcid.org/0000-0003-4477-131X

\section{Lina Facia Izurieta Mena}

ORCID: https://orcid.org/0000-0002-0187-7563

\section{Rosario Paredes}

ORCID: https://orcid.org/0000-0002-7640-4525

Universidad de las Fuerzas Amadas ESPE (Ecuador )

* Licenciada en Ciencias de la Educación Inicial. Universidad de las Fuerzas Armadas ESPE. Ecuador paola.15tw@gmail.com ** Licenciada en Psicología. Master en Discapacidad y Dependencia. Docente de la Universidad de las Fuerzas Armadas ESPE. Ecuador. alijaqui@hotmail.com

*** Licenciada en Ciencias de la Educación. Magister en Proyectos Educativos y Sociales. Docente Tiempo Parcial de la Universidad de las Fuerzas Armadas ESPE. Ecuador. 1fizurieta@espe.edu.ec

*** Licenciada en Ciencias de la Educación mención Educación Básica. Magister en Pedagogía. Ecuador. rlparedes2@espe.edu.ec

\section{Recibido:}

15-01-2021

Aceptado:

05-04-2021
THE CHILDREN'S STORY TO ENHANCE LANGUAGE IN CHILDREN WITH AUTISM 

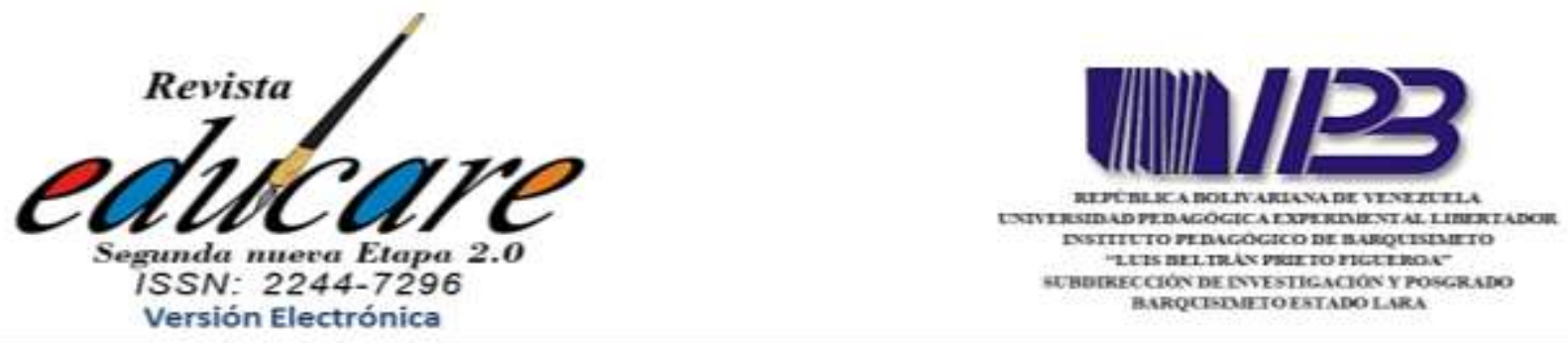

\section{Resumen}

Uno de los principales retos en los niños con trastorno del espectro autista (TEA) es el desarrollo adecuado de su competencia comunicativa con sus pares y adultos. En este trabajo, se realizó un estudio descriptivo de carácter documental, en el cual se aborda al uso del cuento infantil con este propósito. Para esto, se seleccionó sistemáticamente la literatura científica en diferentes bases de datos entre los años 2000 y 2020, centrándose en criterios como el número de artículos publicados por año, áreas académicas y países en donde se investiga con mayor énfasis este tema, así como la literatura infantil dirigida a niños con TEA. Los resultados encontrados más relevantes indican que el uso de los cuentos convencionales es efectivo si se realiza una adaptación pertinente, recurriendo a técnicas que beneficien la comprensión del texto, ya sea mediante el uso de pictogramas, métodos de lectura y otros materiales físicos o tecnológicos.

Palabras clave: cuento; lenguaje; trastorno del espectro autista (TEA)..

\section{Abstract}

One of the main challenges in children with autism spectrum disorder (ASD) is the adequate development of their communicative competence with their peers and adults. In this work, a descriptive documentary study was carried out, in which the use of children's stories for this purpose is addressed. For this, the scientific literature was systematically selected in different databases between the years 2000 and 2020, focusing on criteria such as the number of articles published per year, academic areas and countries where this topic is investigated with greater emphasis, as well as the children's literature aimed at children with ASD. The most relevant results found indicate that the use of conventional stories is effective if a pertinent adaptation is made, resorting to techniques that benefit the understanding of the text, either through the use of pictograms, reading methods and other physical or technological materials.

Keywords: short story; language; autism spectrum disorder (ASD). 


\section{Introducción}

Datos preliminares de la Organización Mundial de la Salud (2019), muestran que 1 de cada 160 niños tiene trastornos del espectro autista (TEA), mientras que Chávez López y Larrea Castelo (2017) indican que, según un informe del Ministerio de Salud Pública del Ecuador de dicho año, 1266 personas presentan estas notables características, con un diagnóstico confirmado en 792 niños. A nivel Latinoamericano, el autismo ha tomado mayor importancia recientemente durante los últimos años; por lo que DiStefano et al. (2016) manifiestan que el $30 \%$ de las personas con trastornos del espectro autista tienen mayores problemas verbales, ya sea en la producción del lenguaje o comprensión del mismo (Eigsti et al., 2011), incluyendo la repetición de frases fijas.

Al analizar la literatura científica, se encontraron diversos aportes sobre distintas estrategias y herramientas para promover la mejora del lenguaje en niños con TEA. Por ejemplo, en la investigación bibliográfica realizada por Carmona et al. (2010) se identificaron 567 trabajos sobre autismo publicados en revistas por autores españoles del año 1974 al 2007 de los cuales solo el 10\% se refieren a Lenguaje y Comunicación. de Carvalho Silva \& Orlando (2019) realizaron un análisis bibliométrico de la producción científica nacional de tesis, disertaciones y artículos relacionando la Danza y el Autismo entre los años 2013 y 2015, sugiriendo la eficacia de la danza como intervención y estimulación en el desarrollo global de niños con autismo.

Asimismo, Lledó, Lorenzo-Lledó, Carreres, \& Vázquez (2020) realizaron un análisis de la producción científica sobre Realidad Virtual (RV) y de las personas con Trastorno del Espectro Autista TEA, considerando que la tecnología es un elemento integrador que puede contribuir a la inclusión de las personas con TEA. Por su parte, Qian (2012) realizó un análisis estadístico de la literatura de investigación en China sobre la barrera del lenguaje en los niños autistas desde los aspectos relacionados con la distribución por edad, de revistas, de escritores y de ángulos de investigación.

Un estudio interesante desarrollado por Suhong (2011), dio a conocer como en el Gray Center for Social Learning and Understanding en Grand Rapids, Michigan, se desarrollaron estrategias basadas en tiras cómicas para ayudar a los niños con autismo en su interacción social. Por último, Vaiouli (2018) realizó una revisión sobre la literatura actual 
sobre el uso sistemático de la música y las intervenciones de musicoterapia como estrategias efectivas para el desarrollo del lenguaje y las habilidades de comunicación preverbal y verbal en niños pequeños con trastornos del espectro autista.

Considerando lo anterior, en este trabajo se presenta una revisión bibliográfica sobre estudios que relacionan específicamente al uso del cuento como una estrategia para mejorar el lenguaje de un niño que presente TEA. Para esto, se plantearon las siguientes preguntas: ¿Cuántas investigaciones existen respecto al tema planteado?, ¿En qué área y país se publican más estudios en cuanto a este tema?, ¿Cuál es el aporte del cuento infantil para potenciar el lenguaje en niños con TEA? y, ¿Existe literatura infantil dirigida a niños con autismo?

\section{Argumentación teórica}

El trastorno de espectro autista (TEA) es una condición que presenta entre otras dificultades, una serie de rasgos o peculiaridades en el habla, ya sea la ecolalia (repetición de frases o palabras emitidas por el emisor), empleo de neologismos o expresiones aparentemente irrelevantes, lo cual es trasladado a problemas de comunicación que influye directamente en la limitación de interacción social (Belinchón \& Rivière, 2017). Se incluye, además, los problemas conductuales estereotipados y una restringida variedad de intereses (Bravo Oro et al., 2012). En concordancia con lo anterior, investigaciones realizadas desde épocas anteriores focalizadas en el déficit de comunicación, permitieron establecer a este limitante como una de las primeras características para predecir la presencia de este trastorno (Kjelgaard\&Tager-Flusberg, 2001)

Por otra parte, Payuelo (2003, citado en Saldaña Monago, 2012), menciona que el lenguaje verbal se direcciona a una conducta expresiva, el cual ejerce una función cognitiva y social que se realiza a lo largo de la vida del ser humano. Igualmente, Glöckler et al. (2006) mencionan que el proceso del desarrollo del lenguaje incrementa cuando existe movimiento corporal, puesto que adquieren experiencias activas y anímicas para la adquisición de nuevo vocabulario y pensamiento. A la par, según Bus, van Ijzendoorn, \&Pelligrini; Newton, Padak\&Rasinski; Christ\& Wang (1995, 2008, 2010, citados en McKenzie, 2014) los docentes de preescolar utilizan varias estrategias orientadas en el desarrollo del vocabulario, por medio de una práctica habitual y eficaz para introducir nuevo léxico a los niños de preescolar a partir de la lectura de cuentos. 
El cuento infantil es un recurso educativo interdisciplinar que proporciona la amplitud para desarrollar diferentes destrezas en cuanto a la comprensión y expresión del lenguaje (Pérez et al., 2013), además del aporte de elementos lingüísticos y paralingüísticos que estos abarcan al momento de ser relatados (Saldaña Monago, 2012). Conjuntamente, presenta un elevado beneficio, en cuanto a la comúnmente conocida "lectura en voz alta", ya que presenta a los niños un amplio repertorio de vocabulario nuevo, cuando estos son escuchados (McKenzie, 2014). Por ejemplo, el en el estudio de Wong \& So (2018) se obtiene que los niños de seis a doce años manifestaron que a partir de la aplicación de la lectura narrada se refuerza el estado gestual del infante, y que a futuro será autónomo y tendrá efecto en una intención comunicativa.

\section{Aspectos metodológicos}

La metodología que se abordó en la presente investigación corresponde a un enfoque cualitativo en el cual se realizó una revisión sistemática de la literatura científica, que se define como un estudio descriptivo de carácter documental, selectivo que presenta la integración de varias perspectivas conforme al tema abordado, y que contiene la selección de investigaciones relevantes (Carrasco, 2009). Para esta finalidad se determinaron ciertas categorías de análisis correspondientes a: Cantidad y evolución de estudios en esta área, procedencia geográfica y científica de dichos estudios, y principales aportaciones encontradas en este tipo de estudios.

\section{Procedimiento de recolección de información}

Para la selección de la literatura se consideraron varios criterios, que permitieron encontrar información relacionada a artículos indexados en español e inglés, y limitando la búsqueda a partir del año 2000 hasta el 2020. En la tabla 1 se definen los criterios de selección y exclusión utilizados:

\section{Tabla 1}

Criterios de selección y exclusión

\begin{tabular}{|l|l}
\hline CRITERIOS DE & $\begin{array}{l}\text { Estudios por su relevancia } \\
\text { SELECCIÓN }\end{array}$ \\
& Estudios enfocados en Medicina, Psicología y Educación \\
& Estudios entre el 2000 y 2020 \\
& Estudios de formato textual \\
& Estudios descriptivos con aportes extras
\end{tabular}




\begin{tabular}{|l|l|}
\hline & $\begin{array}{l}\text { Página de autor corporativo y tesis } \\
\text { Estudios con autor } \\
\text { Estudios con año de publicación } \\
\text { Estudios de varios idiomas } \\
\text { Estudios asequibles }\end{array}$ \\
\hline CRITERIO DE & $\begin{array}{l}\text { Estudios poco citados } \\
\text { Estudios legislativos, biográficos. } \\
\text { Extudios anteriores al 2000 } \\
\text { Estudios de formato multimedia, sonoros. }\end{array}$ \\
\hline
\end{tabular}

\section{Bases de datos y ecuaciones de búsqueda}

En cuanto a la obtención de la información se realizó una búsqueda sistemática a partir de los siguientes buscadores: Scopus, Science Direct, ERIC, Scielo, Dialnet, Google Scholar. Así también, se identificó las palabras clave para ubicar la literatura (Creswell John W., 2014). Por lo tanto, a partir de estas ideas clave se efectuaron 19 ecuaciones de búsqueda con una totalidad de 13 términos individuales, definidos en tres categorías generales: A* =relación entre el desarrollo del lenguaje y autismo; $\mathrm{B}^{*}=$ relación entre el cuento y autismo; $\mathrm{C}^{*}=$ relación entre el desarrollo del lenguaje y el cuento. Las comillas se utilizaron para definir los términos más concretos el tema abordado, el código “AND” o “+” se utilizó para agregar dos temas y realizar una conjunción, usados respectivamente en la búsqueda del idioma inglés y del idioma español, de la misma manera el término "OR" para indicar al motor de búsqueda que puede seleccionar un término u otro.

\section{Tabla 2}

\section{Términos de búsqueda}

\begin{tabular}{|c|c|c|}
\hline $\begin{array}{c}\text { A1. "desarrollo del lenguaje" } \\
+ \text { autismo }\end{array}$ & B1. "cuento" +" autismo" & $\begin{array}{c}\text { C1. "language development } \\
\text { AND "children's story" }\end{array}$ \\
\hline $\begin{array}{c}\text { A2. "language development" } \\
\text { AND "autism" }\end{array}$ & $\begin{array}{c}\text { B2. "children's story" AND } \\
\text { "autism" }\end{array}$ & $\begin{array}{c}\text { C2. "desarrollo del lenguaje" } \\
+" \text { cuento infantil" }\end{array}$ \\
\hline A3. "language" + "autism" \\
OR "TEA" & $\begin{array}{c}\text { B3. "cuento infantil" AND } \\
\text { "autismo" OR "trastorno del } \\
\text { espectro autista" }\end{array}$ & $\begin{array}{c}\text { C3. "cuento" OR "cuento } \\
\text { infantil" AND "desarrollo del } \\
\text { lenguaje" }\end{array}$ \\
\hline
\end{tabular}


El cuento infantil como estrategia para potenciar el lenguaje en niños con autismo

Guanoluisa Deisy Paola; Álvarez Mérida Alicia Jaqueline; Izurieta Mena Lina Facia, Paredes Morales Rosario de Lourdes\& Paredes Rosario

\begin{tabular}{|c|c|c|}
\hline A4. "autismo" +"lenguaje" & $\begin{array}{c}\text { B4. "literatura infantil" } \\
+" \text { autismo" }\end{array}$ & $\begin{array}{c}\text { C4. storybook reading AND } \\
\text { vocabulary }\end{array}$ \\
\hline A5. comunicación +"autismo" & $\begin{array}{c}\text { B5. "storybook reading" } \\
\text { AND autism }\end{array}$ & $\begin{array}{c}\text { C5. storybook reading AND } \\
\text { communication }\end{array}$ \\
\hline $\begin{array}{c}\text { A6. "lenguaje verbal y no } \\
\text { verbal" +"autismo" }\end{array}$ & $\begin{array}{c}\text { B6. story reading AND } \\
\text { autism }\end{array}$ & \\
\hline $\begin{array}{c}\text { A7. "comunication" } \\
\text { AND"autism" }\end{array}$ & & \\
\hline A8. vocabulary AND autism & & \\
\hline
\end{tabular}

\section{Gestión de los resultados de la búsqueda}

A partir de las ecuaciones de búsqueda se realizó la selección y sistematización de los artículos obtenidos a través de bases de datos consideradas (tabla 3), para posteriormente ser consolidadas utilizando el gestor bibliográfico Mendeley. A continuación, se procesaron estos estudios mediante el software DOCEAR, en el cual se organizaron los mismos por idioma, año, y bases de datos utilizadas, además se excluyó estudios que no completaban los criterios de inclusión. Para la síntesis de la literatura se realizó una bitácora en el software Excel con filtros determinados en cuanto a la problemática abordada, enfoque investigativo, resultados y discusión.

\section{Tabla 3}

Resultados de documentos obtenidos de manera general

\section{BASE DE DATOS Resultados (documentos en general)}

$\begin{array}{ll}\text { Scopus } & 31 \\ \text { Science Direct } & 58 \\ \text { ERIC } & 65 \\ \text { Scielo } & 26 \\ \text { Dialnet } & 18 \\ \text { Google Académico } & 25\end{array}$

\section{Presentación de los resultados}

Luego de recabar la información sobre la literatura encontrada utilizando los criterios de búsqueda presentados anteriormente, se identificaron los siguientes elementos o criterios 
correspondientes a las categorías de análisis inicialmente planteadas: Respecto a la cantidad y evolución de estudios, se determinó el total de publicaciones por año.

Sobre la procedencia geográfica y científica se identificó el área científica y el país de donde provienen cada estudio. Respecto a las principales aportaciones encontradas, se analizó en ciertos casos las contribuciones específicas que brindó el uso del cuento infantil para potenciar el lenguaje en niños con TEA, y en otros casos se identificó la elaboración de literatura científica dirigida a esta población de interés.

Con base en lo anterior, los resultados de la presente revisión se exponen de la siguiente manera: número de publicaciones entre el año 2000 y 2020; área académica donde más se publica sobre el tema; país donde se han realizado más estudios; aporte del cuento infantil para potenciar el lenguaje en los niños con autismo; Literatura infantil dirigida a la población con autismo.

\section{Número de publicaciones entre el año 2000 y 2020}

Los estudios analizados entre 2000 y 2020 que cumplieron con los criterios de selección se presentan en la siguiente figura, siendo este un porcentaje minoritario de estudios enfocados a las variables del cuento infantil y TEA.

\section{Figura 1}

\section{Documentos publicados por año}

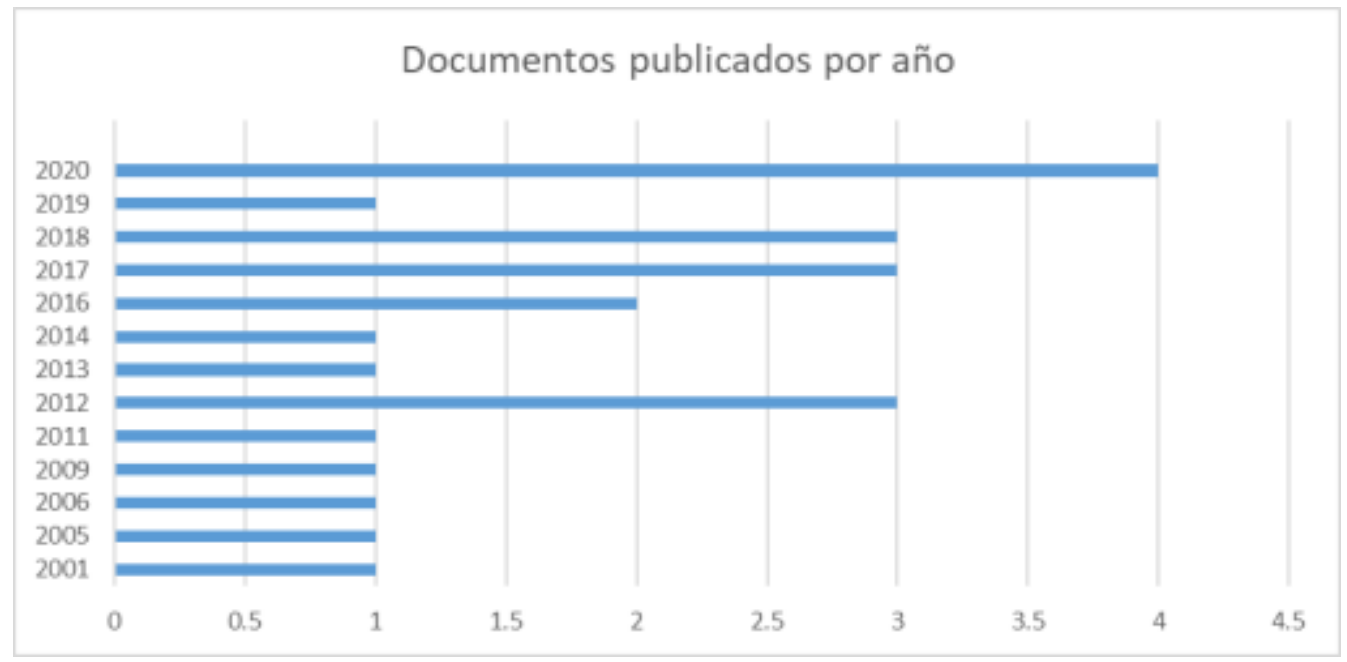

\section{Análisis por Áreas académicas y por países}

El área donde más se realizó investigaciones conforme a las variables, entre el año 2000 y 2020, es de Ciencias de la Educación. Un aspecto fundamental dentro de estas ciencias 
está enfocados al rol del docente como mediador del proceso de aprendizaje en niños con TEA. Como ejemplo, Reuven Feuerstein (citado en Noguez Casado, 2002) en su teoría de la modificabilidad cognitiva, recalca la importancia la acción de un mediador o docente para mejorar el rendimiento académico y social en niños con autismo.

\section{Tabla 4}

Documentos publicados por área

\begin{tabular}{lcc}
\hline Área & $\begin{array}{c}\text { Número de } \\
\text { documentos }\end{array}$ & Porcentaje \\
\hline Ciencias de la Educación & 8 & $35 \%$ \\
Neurociencia & 6 & $26 \%$ \\
Psicología & 5 & $22 \%$ \\
Medicina & 4 & $17 \%$
\end{tabular}

Nota: En la tabla 4 se presentan los resultados en esta y otras áreas consideradas

Estos resultados indican además que los materiales publicados en los últimos 20 años son 23 , con una media de 1,8 artículos por año repartidas en diferentes ciencias, pero en cuanto a las Ciencias de la Educación son 8, los cuales representan el 35\% del total, por lo que los campos restantes como neurociencia, psicología y medicina predominan en atender a esta población.

\section{Análisis por países}

En cuanto al país con más estudios publicados entre el año 2000 y 2020, tomado de igual manera de las variables cuento infantil y autismo, Estados Unidos es el que lleva un mayor abordaje del tema; seguido se encuentra España como segundo país en aportar estudios a esta población con TEA 


\section{Figura 2}

Documentos publicados por país

\section{Documentos publicados por país}

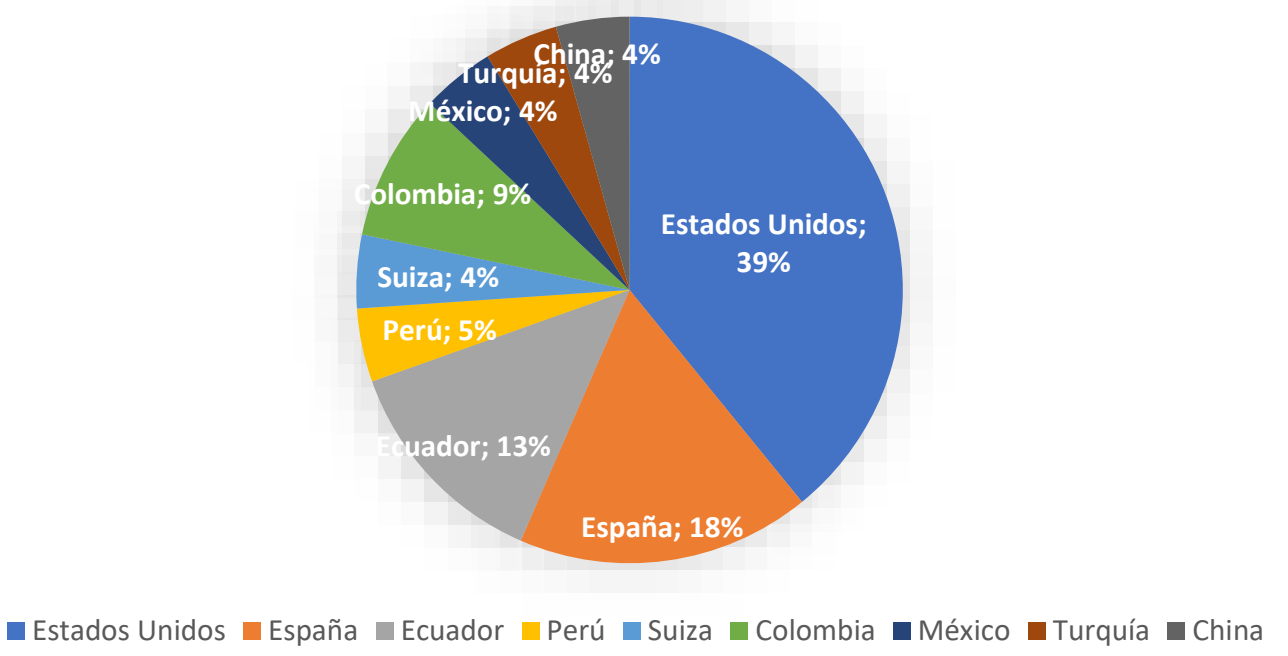

Cabe mencionar además que, la sumatoria del porcentaje de los países de América Latina, el 30\% corresponde al porcentaje de estudios que aporten al tema, siendo este una cantidad baja de aportes para esta población a comparación del porcentaje entre España y Estados Unidos que cubren el 57\%.

\section{Aportes del cuento infantil para potenciar el lenguaje en niños con TEA}

Los cuentos cumplen un papel importante como parte del crecimiento de los seres humanos, el cual permite reinventar y crear personajes, jugar con la imaginación, construir nuevos espacios o ambientes de hechos, el cual conlleva al aprendizaje sin excluir al niño de su entorno inmediato. Los niños con TEA presentan dificultad en la comunicación la cual afecta en su interacción social, por lo que los cuentos les ayudan a mejorar las habilidades lingüísticas, lo que les permite comprender la información que se les proporciona y potenciar su socialización.

Dentro de las varias aportaciones que se encontraron en las revisiones bibliográficas respecto a este tema, sean encontrado las siguientes:

- Uso de Tecnología: Al fomentar la lectura interactiva de cuentos con dispositivos electrónicos, pantallas, páginas web y otros recursos 
audiovisuales, esto permite que el niño con TEA asimile palabras abstractas y adquiera nuevo vocabulario (Alison et al., 2017).

- Uso de pictogramas: Los cuentos con pictogramas implican la utilización de la doble codificación, icónica y verbal, además que dichos pictogramas sustituyen a una palabra en la narración. Así también, los pictogramas son el primer acercamiento a la lectura (Segovia Vázquez, 2016).

- Intervención del mediador en cuanto a la animación a la lectura: Permite mejorar la expresión del lenguaje, gracias al apoyo de una serie de actividades de narración, debido a que la voz, entonación y recursos visuales que el docente utiliza, permite que el niño comience a disfrutar del proceso lector (Kim et al., 2018). Asimismo, el docente tiene la posibilidad de enriquecer la imaginación y de orientar sus estrategias de enseñanza de acuerdo con las necesidades de sus estudiantes. Es muy importante ya que se prepara con anticipación sobre la lectura del cuento, mientras que durante la lectura es necesario reanimar los hechos para verificar la comprensión de la lectura y finalmente, es después de la lectura cuando se realiza una mini prueba, cambiando personajes o hechos, además de desarrollar la comunicación entre el infante y el lector. Además, según Soares et al. (2020) resaltan que la guía continua del docente es de vital importancia para que los niños logren comprender el texto y así mismo es sustancial el acompañamiento directo como mediador para el aprendizaje de vocabulario.

- Logros alcanzados: Bean et al. (2020) mencionan que los niños con autismo pueden llegar a tener un interés autónomo por la lectura de libros. Cuando se aplica la lectura de cuentos en educación infantil como técnica de alfabetización, se toma en cuenta que pueden intervenir para deletrear, contar, recordar, resolver acertijos, y por tanto ayuda que los niños con características del trastorno del espectro autista practiquen y mejoren sus habilidades lingüísticas, y de igual manera como estrategia para que en un futuro el discente tenga una lectura eficaz e independiente (Sani-Bozkurt et al., 2020). 
El cuento infantil como estrategia para potenciar el lenguaje en niños con autismo

Guanoluisa Deisy Paola; Álvarez Mérida Alicia Jaqueline; Izurieta Mena Lina Facia, Paredes Morales Rosario de Lourdes\& Paredes Rosario

\section{Literatura infantil creada para niños con autismo}

De acuerdo a Segovia Vázquez (2016) los cuentos dirigidos para niños con autismo fueron creados a partir de la necesidad de comprender o desarrollar el lenguaje en niños con autismo de manera interactiva, por consiguiente, se extrajo la lista de cuentos adaptados para niños con la condición del autismo. De lo revisado apenas son 13 cuentos publicados en 10 años, destacándose España con 13 publicaciones.

\section{Tabla 5}

Lista de cuentos dirigidos a niños con autismo

\begin{tabular}{|c|c|c|c|c|c|}
\hline & Autor & Año & Título & Ciudad/País & Editorial \\
\hline 1 & Monreal Díaz, V & 2002 & SOS se necesita sonrisa & Madrid & Everest \\
\hline 2 & Oro, B. & 2003 & El elefanta Jo & $\begin{array}{l}\text { Boadilla del } \\
\text { Monte (Madrid) }\end{array}$ & SM \\
\hline 3 & Villán, O. & 2004 & El conejo blanco & Pontevedra: & Kalandraka. \\
\hline 4 & Núñez, L. (2005). & 2005 & $\begin{array}{l}\text { El largo viaje de los } \\
\text { dragones }\end{array}$ & Madrid & Alfaguara \\
\hline 5 & Reviejo, C. & 2005 & Abezoo. & $\begin{array}{l}\text { Boadilla del } \\
\text { Monte (Madrid) }\end{array}$ & SM \\
\hline 6 & Suárez Otero, A. & 2010 & $\begin{array}{l}\text { - Nicolás cocina sin fuego } \\
\text { - Nicolás va de compras }\end{array}$ & Sevilla & Kalandraka \\
\hline 7 & Carrillo, M.L. & 2011 & Pablo tiene un hermano & Madrid & $\begin{array}{l}\text { Ciencias de la } \\
\text { Educación } \\
\text { Preescolar y } \\
\text { Especial, D.L. }\end{array}$ \\
\hline 8 & Bonavista, R. & 2012 & $\begin{array}{l}\text { La fantástica historia de } \\
\text { Caperucita Roja }\end{array}$ & Barcelona & Beascoa \\
\hline 9 & Calvo Llorente, A. & 2012 & Ana y la navidad & Granada & GEU, D.L \\
\hline 10 & Garrigós, A. & 2013 & $\begin{array}{l}\text { La tortuga Filomena no } \\
\text { sabe hacer amigos. }\end{array}$ & Valencia & Psylicom, D.L. \\
\hline 11 & Monreal, V. & 2014 & $\begin{array}{l}\text { Cuentos de colores de } \\
\text { princesas }\end{array}$ & Madrid & BRUÑO \\
\hline 12 & Sánchez, M.J. & 2015 & $\begin{array}{l}\text { Las fantásticas aventuras de } \\
\text { tres amigos y Pico }\end{array}$ & Barcelona & Alfaguara \\
\hline 13 & Reyes, M. & 2015 & $\begin{array}{l}\text { Las pelusas se mudan de } \\
\text { casa }\end{array}$ & España & $\begin{array}{l}\text { Aprendices } \\
\text { visuales. } \\
\text { www.aprendices } \\
\text { visuales.com }\end{array}$ \\
\hline
\end{tabular}

La orientación general de los cuentos anexados se basa en el abordaje de acciones y sucesos cotidianos, de manera que el niño con TEA comprenda conceptos o léxico mediante los cuentos, puesto que, al adquirir vocabulario, el infante con trastorno del espectro autista 
El cuento infantil como estrategia para potenciar el lenguaje en niños con autismo

Guanoluisa Deisy Paola; Álvarez Mérida Alicia Jaqueline; Izurieta Mena Lina Facia, Paredes Morales Rosario de Lourdes\& Paredes Rosario

relacione con la cotidianidad y así léxico adquirido se transforme en intención comunicativa, por lo que se potenciará el lenguaje verbal.

\section{Conclusiones y recomendaciones}

El autismo es un trastorno aún desconocido y los estudios abordados en el presente artículo en su mayoría, fueron de un idioma diferente al español, debido a la escasa investigación sobre el autismo y el desarrollo del lenguaje. Esto también se evidencia al estudiar la relación del uso del cuento en niños con TEA, aunque se conozca del importante impacto que tiene esta estrategia en la comunicación y el desarrollo del lenguaje. A consecuencia de esto y de factores que no son motivo de esta investigación, la literatura dirigida a esta población también es escasa.

El cuento infantil como recurso interdisciplinario, ayuda de manera secuencial en la adquisición de vocabulario y trasladado a lenguaje para comunicar ideas o sentimientos con las personas de su entorno, así como afirma Sandoval Paz (2005). Es a través de este recurso que los niños recrean acciones relatadas y se integran al mundo que los rodea.

El uso de pictogramas en los cuentos es una herramienta innovadora que capta la atención de los niños. Al respecto, Casa et al. (2018) mencionan que esto les permite generar la conceptualización. Corroborando con Chamba-Rueda \& Chillogallo-Ordóñez (2019), el cuento permite que los niños estructuren oraciones, que posteriormente son comunicadas de manera oral, además del incentivo de la creatividad y de la interacción social.

Se considera que, al incrementar el lenguaje como primer eslabón, se puede trasladar posteriormente a una intención comunicativa y a un mejor desarrollo de interacción (ArtigasPallares \& Paula, 2012; Bacon et al., 2019), de allí la importancia de desarrollar el lenguaje en los primeros años de vida del niño, aún más en los niños con autismo.

Así también, el aporte de un mediador como es el docente permite que el aprendizaje sea correspondido por el infante, dado que será quien del significado a los recursos y ambientes en donde se sumerge el niño con TEA, para que de esta manera el infante adquiera un mayor vocabulario gracias al uso del cuento y a su vez se eleve su intención comunicativa.

Finalmente, la limitación del estudio radica en la escasa investigación a nivel Latinoamericano en cuanto al tema abordado. Los resultados obtenidos son de utilidad ya que hay un $30 \%$ de personas con esta condición del trastorno del espectro autista (DiStefano et al., 2016). Queda la puerta abierta para realizar estudios del área Educativa, que permitan 
analizar los beneficios y resultados inducidos a partir el uso del cuento como estrategia para potenciar el lenguaje en este grupo de personas.

\section{Referencias}

Alison, C., Root, J. R., Browder, D. M., \& Wood, L. (2017). Technology-Based Shared Story Reading for Students With Autism Who Are English-Language Learners. Journal of Special Education Technology, 32(2), 91-101. https://doi.org/10.1177/0162643417690606

Artigas-Pallares, J., \& Paula, I. (2012). El autismo 70 años después de Leo Kanner y Hans Asperger. Revista de La Asociación Española de Neuropsiquiatría, 32(115), 567587. https://doi.org/10.4321/s0211-57352012000300008

Bacon, E. C., Osuna, S., Courchesne, E., \& Pierce, K. (2019). Naturalistic language sampling to characterize the language abilities of 3-year-olds with autism spectrum disorder. Autism, 23(3), 699-712. https://doi.org/10.1177/1362361318766241

Bean, A. F., Perez, B. I., Dynia, J. M., Kaderavek, J. N., \& Justice, L. M. (2020). BookReading Engagement in Children with Autism and Language Impairment: Associations with Emergent-Literacy Skills. Journal of Autism and Developmental Disorders, 50(3), 1018-1030. https://doi.org/10.1007/s10803-019-04306-4

Belinchón, M., \& Rivière, Á. (2017). El lenguaje autista desde una perspectiva correlacional.

9395(April). https://doi.org/http://dx.doi.org/10.1174/021093900320380695

Bravo Oro, A., Vázquez Briseño, J., Cuello García, C. A., Calderón Sepúlveda, R. F., Hernández Villalobos, A. M., \&Esmer Sánchez, C. (2012). Early manifestations of autism spectrum disorders. Experience of 393 cases in a child neurological centre. Neurología (English Edition), 27(7), 414-420. https://doi.org/10.1016/j.nrleng.2011.09.002

Carmona, M. B., Muñoz, L. B., De Andrés, E. G., Biggi, J. F., \& De La Paz, M. P. (2010). Evolución de los estudios sobre autismo en España: publicaciones y redes de $\begin{array}{lllll}\text { coautoría entre } 1974 \quad \text { y } 2007 . & \text { Psicothema, 242-249 }\end{array}$ https://www.redalyc.org/articulo.oa?id=72712496011

Carrasco, O. V. (2009). CÓMO ESCRIBIR ARTÍCULOS DE REVISIÓN. Recuperado el 18 de 08 de 2020, de Revista Médica La Paz, 15(1), 63-69.: 
http://www.scielo.org.bo/scielo.php?script=sci_arttext\&pid=S1726-

$89582009000100010 \& \operatorname{lng}=$ es \& tlng=es.

Casa, F., Clavijo, H., González-Moreno, C. X., Jiménez Ortíz, M. L., Gordo Contreras, A., Casas Rustarazo, R., Andrés-Roqueta, C., Baixauli-fortea, I., Gómez-garcía, S., Puig, M. D. El, Oliver, J., Chamba-Rueda, L. P., Chillogallo-Ordóñez, J. E., Martha, J., Aurora, G., Pérez, R., Mural, V., Hervás Zúñiga, A., Balmaña, N., ... ع ع ا. (2018). El cuento infantil: facilitador de pensamiento desde una experiencia pedagógica. Praxis \& Saber, 1(2), 100. https://doi.org/10.19053/22160159.3027

Chamba-Rueda, L. P., \& Chillogallo-Ordóñez, J. E. (2019). El cuento infantil en el desarrollo del lenguaje oral en niños del primer grado de educación general básica. Dominio de Las Ciencias, 5(3), 697. https://doi.org/10.23857/dc.v5i3.959

Chávez López, C., \& Larrea Castelo, M. de L. (2017). Autismo en Ecuador: Un grupo social en espera de atención. Revista Ecuatoriana de Neurologia, 26(3), 203-214. http://revecuatneurol.com/wp-content/uploads/2018/03/Autismo-en-Ecuador.-

Autism-in-Ecuador.pdf

Creswell John W. (2014). Research-Design_Qualitative-Quantitative-and-Mixed-MethodsApproaches (4 Edition). http://fe.unj.ac.id/wp-content/uploads/2019/08/ResearchDesign_Qualitative-Quantitative-and-Mixed-Methods-Approaches.pdf de Carvalho Silva, E., \& Orlando, R. M. (2019). A interface dança e autismo: o que nos revela a produção científica. Revista Educação Especial, 61-1. https://doi.org/10.5902/1984686X33121

DiStefano, C., Shih, W., Kaiser, A., Landa, R., \&Kasari, C. (2016). Communication growth in minimally verbal children with ASD: The importance of interaction. Autism Research, 9(10), 1093-1102. https://doi.org/10.1002/aur.1594

Eigsti, I. M., De Marchena, A. B., Schuh, J. M., \& Kelley, E. (2011). Language acquisition in autism spectrum disorders: A developmental review. Research in Autism Spectrum Disorders, 5(2), 681-691. https://doi.org/10.1016/j.rasd.2010.09.001

Glöckler, M., Langhammer, S., \&Wiechert, C. (2006). Salud a través de la Educación. Iraola, E. G. (2015). Los beneficios de la lectura compartida de libros: breve revisión. Educación XX1, 303-324 https://doi.org/ 10.5944/educXX1.18.1.12334 
Kim, S. Y., Rispoli, M., Lory, C., Gregori, E., \& Brodhead, M. T. (2018). The Effects of a Shared Reading Intervention on Narrative Story Comprehension and Task Engagement of Students with Autism Spectrum Disorder. Journal of Autism and Developmental Disorders, 48(10), 3608-3622. https://doi.org/10.1007/s10803-018$3633-7$

Kjelgaard, M. M., \&Tager-Flusberg, H. (2001). An investigation of language impairment in autism: Implications for genetic subgroups. Language and CognitiveProcesses, 16(23), 287-308. https://doi.org/10.1080/01690960042000058

Lledó, G. L., Lorenzo-Lledó, A., Carreres, A. L., \& Vázquez, E. P. (2020). Enfoque desde una perspectiva bibliométrica de la aplicación educativa de la realidad virtual en personas con Trastorno del Espectro Autista. Education in the knowledge society $(E K S), 5$

McKenzie, E. (2014). Vocabulary Development Using Visual Displays. Dimensions of Early Childhood, $42(2)$, $12-17$. http://proxygw.wrlc.org/login?url=http://search.ebscohost.com/login.aspx?direct=tr $\mathrm{ue} \& \mathrm{db}=$ eric $\& \mathrm{AN}=\mathrm{EJ} 1044052 \&$ site $=$ ehost live\%5Cnhttp://www.southernearlychildhood.org/publications.php

Mera Segovia, C. M., \&Berlis, G. L. (2020). Neurofunciones en la enseñanza preescolar: importancia en el proceso de enseñanza aprendizaje y la atención de salud. $24(\mathrm{CCM})$.

Noguez Casado, S. (2002). El desarrollo del potencial de aprendizaje: Entrevista a Reuven Feuerstein. El Desarrollo Del Potencial de Aprendizaje: Entrevista a Reuven Feuerstein, 4(2), 01-15. http://www.scielo.org.mx/pdf/redie/v4n2/v4n2a9.pdf Organización Mundial de la Salud. (07 de 11 de 2019). Trastornos del espectro autista. Recuperado el 28 de 07 de 2020, de Sitio Web mundial: https://www.who.int/es/news-room/fact-sheets/detail/autism-spectrum-disorders

Pérez, D., Pérez, A. I., \& Sánchez, R. (2013). El cuento como recurso didáctico. 3Ciencias, 1-29. http://dialnet.unirioja.es/descarga/articulo/4817922.pdf

Qian, M. (2012). A Review on Language Barrier of Autistic Children Based on Bibliometric Analysis of Literature. Journal of Suihua University 
Saldaña Monago, R. del P. (2012). Programa de cuentos pictográficos para incrementar el lenguaje oral en niños (as) de 3 años de una I.E. del Callao. http://repositorio.usil.edu.pe/bitstream/123456789/1319/1/2012_Saldaña_Programade-cuentos-pictográficos-para-incrementar-el-lenguaje-oral-en-niñas-de-3-años-deuna-institución-educativa-del-Callao.pdf

Sandoval Paz, C. E. (2005). El cuento infantil: Una experiencia de lenguaje integral. Revista IeRed: Revista Electrónica de La Red de Investigación Educativa, 1(2), 1-9. http://revista.iered.org/v1n2/pdf/csandoval.pdf

Sani-Bozkurt, S., Vuran, S., \&Akbulut, Y. (2020). Design and Use of Interactive Social Stories for Children with Autism Spectrum Disorder (ASD). Contemporary Educational Technology, 8(1), 1-25. https://doi.org/10.30935/cedtech/6184

Segovia Vázquez, C. (2016). Cuentos y autismo. https://idus.us.es/xmlui/handle/11441/45058

Soares, E., Miller, T., Ciffone, K., \& Read, K. (2020). E-books for children with autism: Best read alone or with a therapist? Child Language Teaching and Therapy, 36(1), 19-32. https://doi.org/10.1177/0265659020903795

Suhong, Z. Y. (2011). A Review of the Application of Comic Strip Conversation to Children with Autism. Journal of Nanjing Technical College of Special Education, 11

Vaiouli, P., \&Andreou, G. (2018). Communication and Language Development of Young Children With Autism: A Review of Research in Music. Communication Disorders Quarterly, 39(2), 323-329. https://doi.org/10.1177/1525740117705117

Wong, M. K., \& So, W. (2018). Research in Developmental Disabilities Absence of delay in spontaneous use of gestures in spoken narratives among children with Autism Spectrum Disorders. Research in Developmental Disabilities, 72(January 2016), 128-139. https://doi.org/10.1016/j.ridd.2017.11.004 